\title{
Die Kunst der Kunstbetrachtung: Aspekte einer pragmatischen Ästhetik in Kunstausstellungen
}

\section{Von Dirk vom Lehn}

\section{Einleitung 1}

Die Kunstsoziologie hat sich bisher nur relativ wenig mit der Betrachtung von Kunstwerken beschäftigt. Ihr Hauptinteresse dagegen gilt bislang der Produktion und Institutionalisierung von Kunst sowie der Beziehung von Kunst und Gesellschaftsstruktur (Gerhards 1997; Tanner 2003). Die wenigen Untersuchungen, die sich überhaupt der Wahrnehmung von Kunst zuwenden, fokussieren entweder auf die Beziehung zwischen Wahrnehmung und Kommunikation (Luhmann 1998) oder versuchen die Struktur von Kunstwerken aus der Struktur der Kunstwahrnehmung zu rekonstruieren (Loer 1996). Die Art und Weise, wie Menschen in gewöhnlichen und alltäglichen Situationen in Museen und Galerien Kunstwerke und andere Ausstellungsstücke betrachten, wird von der Soziologie der Kunst jedoch weitgehend ausgespart.

Im Zusammenhang mit den Veränderungen ihrer finanziellen und politischen Rahmenbedingungen zeigen Museen ein wachsendes Interesse an ihren Besuchern. Sie engagieren Besucherforscher, die ein breites Spektrum an Methoden anwenden, um die >Effektivität< von Ausstellungen zu messen (Klein 1996; Schaefer 1996; Schiele 1993). Dabei konzentrieren sie sich zumeist auf die Bildungsfunktion von Museen und versuchen zu zeigen, inwieweit Besucher von Ausstellungen über Kunst, Kultur, Wissenschaft und Technik lernen (Falk/Dierking 2000; Hein 1998). Ihr Fokus auf das Lernen von Besuchern verstellt ihnen jedoch den Blick auf die Handlungs- und Interaktionsprozesse, durch die sich Besucher Ausstellungsstücken zuwenden, um sie zu betrachten und zu verstehen (Lawrence 1993).

Dieser Artikel basiert auf einer Reihe von Forschungsprojekten, in denen wir untersuchen, wie Besucher von Museen und Galerien Ausstellungsstücke inspizieren und erfahren. In vorangegangenen Veröffentlichungen haben wir gezeigt, dass die Art und Weise, wie Besucher Ausstellungsstücke betrachten, benutzen und verstehen, durch soziale Interaktion mit Begleitern und anderen Besuchern beeinflusst und geprägt wird (Heath/vom Lehn 2004; vom Lehn/Heath/Hindmarsh 2001). Dieser Artikel untersucht nun, wie Besucher die sozialen und interaktionalen Bedingungen herstellen, unter denen sie Kunstwerke in Museen betrachten.

\section{Kunstwahrnehmung und Besucherverhalten}

In der Psychologie und den Kognitionswissenschaften gibt es seit langem ein großes Interesse an der Kunstwahrnehmung. Sie vermuten eine Beziehung zwischen der Struktur von Kunstwerken und ihrer Wahrnehmung. Es wird argumentiert, dass Kunstwerke eine besondere Struktur aufweisen, die das Sehen in einer bestimmten Art und Weise stimuliert und beeinflusst (Arnheim 1974; Gombrich 1960; Gombrich 1982). Die Kognitionswissenschaften vermuten den Ursprung dieser Struktur von Kunstwerken neuerdings in der Struktur des menschlichen Gehirns, wobei die kognitiven Strukturen als Basis für die Produktion von

1) Der Artikel ist aus einem Forschungsprojekt hervorgegangen, das vom Arts and Humanities Research Council (AHRC) finanziert wird (Projekt Nummer: APN17441). Ich möchte mich bei meinen Kollegen in der Work, Interaction \& Technology Research Group (King's College London), insbesondere Christian Heath und Karola Pitsch, sowie bei den Gutachtern und Herausgebern der Sozialen Welt für die wertvollen Hinweise hinsichtlich der Analyse der Daten und ihrer Darstellung bedanken. 
Kunstwerken und deren Wahrnehmung angesehen werden (Ramachandran/Hirstein 1999). In diesem Sinne fokussieren Kognitionswissenschaftler auf subjektive und kognitive Prozesse der Kunstwahrnehmung und sind nicht an den praktischen Handlungen und Interaktionen, durch die Museumsbesucher Kunstwerke betrachten und erfahren, interessiert.

Überraschenderweise hat auch die Kunstsoziologie bisher nur relativ wenig Interesse an der Betrachtung von Kunst gezeigt. Sie beschäftigt sich zumeist mit der Produktion von Kunst (Becker 1982) und ihrer Ausstellung (Doering/Hirschauer 1997; Gerhards 1997; Karp/Lavine 1991; Macdonald 2002) sowie mit dem Wesen der Kunst und ihrer gesellschaftlichen Funktion (Luhmann 1998). Untersuchungen, wie die der Rezeptionsästhetik (Warning 1994) oder der Objektiven Hermeneutik (Loer 1996), sind hauptsächlich am Betrachter ${ }^{2}$ interessiert, und nehmen an, dass sie durch ihn etwas über Kunst erfahren können. Die Praktiken der Kunstbetrachtung und ihre Einbettung in die spezifischen Umstände der sozialen Situation, in der sie vollzogen werden, sind in diesem Zusammenhang nur von untergeordneter Bedeutung.

Daneben gibt es die wichtigen Beiträge zur Kunstrezeption von Bourdieu, in denen er herausarbeitet, wie das Verständnis von Kunst mit kulturellen Codes zusammenhängt, die durch Sozialisation und Erziehung erworben werden. Wie Kunst wahrgenommen wird und wie über Kunst kommuniziert wird, hängt - Bourdieu zufolge - einerseits von der Zugehörigkeit zu sozialen Klassen ab und trägt andererseits gleichzeitig zur Reproduktion der Sozialstruktur bei. Obwohl Bourdieus Untersuchungen einen wichtigen Beitrag zu unserem Verständnis der Kunstwahrnehmung leisten, lassen sie die praktische und soziale Organisation der Kunstbetrachtung, wie sie an den Werken selbst vollzogen wird, außer acht (Bourdieu 1987, 1991).

Diese Forschungslücke haben jüngst Besucherforscher adressiert, die Gespräche von Besuchern vor Ausstellungsstücken untersuchen. Indem sie den Inhalt der Gespräche vor den Exponaten mit den Absichten der Museumsmanager und Kuratoren vergleichen, versuchen sie die Effektivität der Informationsvermittlung von Ausstellungen zu evaluieren. Ihre Befunde werden dazu verwendet, die Informationsvermittlung durch Ausstellungsstücke zu verbessern, indem Veränderungen an ihrem Design und ihrer Präsentation vorgenommen werden (Leinhardt/Crowley/Knutson 2002; Leinhardt/Knutson 2004). Die Evaluationen sind weder an der sozialen Organisation der Gespräche vor Ausstellungsstücken noch an ihrer Koordination mit der Betrachtung der Objekte interessiert.

Dies wiederum ist das Thema einiger Untersuchungen in der Gesprächsforschung. Sie haben damit begonnen, die Formen und Funktionen der Kommunikation über Kunstwerke herauszuarbeiten (Hausendorf 2005; Kindt 1982). Allerdings fokussiert die Gesprächsforschung nahezu ausschließlich auf die verbalen Aspekte der Interaktion, während die körperlichen und materialen Handlungen der Besucher sowie deren Koordination mit verbalen Äußerungen unbeachtet bleiben.

Detaillierte video-basierte Untersuchungen wenden sich dieser Forschungslücke zu und leisten einen Beitrag zum Verständnis der sozialen Praktiken der Kunstbetrachtung. Sie können zeigen, wie Besucher ihre Betrachtung und Inspektion von Kunstwerken miteinander koordinieren und ihre Erfahrung von Kunst durch verbale, körperliche und materiale Handlungen und Interaktionen vollziehen.

2) Im Verlauf des Artikels verwenden wir zu Gunsten der Lesbarkeit des Textes durchgehend den maskulinen Genus >Betrachter< und die entsprechenden maskulinen Pronomen, obwohl natürlich, wie auch die gewählten Fragmente belegen, ein großer Teil der Besucher weiblich ist. 


\section{Video-basierte Ethnografie in Museen}

In jüngerer Zeit haben Soziologen zunehmendes Interesse daran gezeigt, visuelles Material wie Fotos, Filme und Videos als Daten zu sammeln und analysieren. Sie haben analytische und methodologische Theorien und Konzepte entwickelt, um adäquat mit diesem Datentyp umzugehen und bilden einzelne Fotos oder >frames< aus Filmen und Videoclips in ihren Publikationen als Nachweis für ihre Befunde ab. In der deutschsprachigen Soziologie sind in diesem Zusammenhang insbesondere gattungsanalytische und hermeneutische Verfahren zur Analyse von Videodaten herangezogen wurden (Raab 2002; Schnettler 2001, 2003). ${ }^{3}$ Der vorliegende Artikel stützt sich dagegen auf Goffmans (1963; 1971) bekannte Untersuchungen zum Verhalten in öffentlichen Räumen, auf Kendons (1990a) video-basierte Analyse von sozialer Interaktion sowie auf neuere Entwicklungen in Ethnomethodologie (Garfinkel 1967) und Konversationsanalyse (Sacks 1992), insbesondere den >Workplace Studies< (Bergmann 2005; Eberle 1997; Heath/Luff 2000; Knoblauch 2004; Knoblauch/Heath 1999; Luff/Hindmarsh/Heath 2000). Die Workplace Studies haben systematisch die soziale Organisation von körperlichen, sprachlichen und materialen Aspekten menschlicher Handlungen herausgearbeitet (Goodwin 1981; Heath 1986). In ihrem Mittelpunkt stehen die Ressourcen und Praktiken, die Interaktionsteilnehmer verwenden, wenn sie soziale Handlungen und Interaktionen vollziehen und den Handlungen anderer Teilnehmer Sinn verleihen. Diese Studien arbeiten systematisch die sequentielle Organisation der Handlungen heraus und explizieren, wie Teilnehmer ihre Handlungen miteinander koordinieren und sich dabei zur materialen Umgebung hin orientieren.

Der besondere Fokus dieses Artikels gilt der Interaktionsordnung in Kunstmuseen und galerien. Es wird danach gefragt, wie Besucher Ausstellungsstücke in und durch Interaktion mit Begleitern und anderen Besuchern, die sich in der gleichen räumlichen Umgebung aufhalten, betrachten und erfahren. Diese Interaktionsordnung in Ausstellungen wird zuweilen als »Museumsdisziplin« (Hirschauer 2002: 10) und als »stummes Geschiebe« (ibid.) entlang der Ausstellungsstücke charakterisiert. Einige Autoren (Bennett 1995; Trondsen 1976) argumentieren, dass die museumsspezifische Ordnung sozialen Handelns durch die Architektur und räumliche Gestaltung von Ausstellungen ermöglicht würde; diese gestalterischen Mittel schafften die Voraussetzungen für eine panoptische Situation, in der »soziale Kontrolle « des Besucherverhaltens durch wechselseitige Beobachtung und Beobachtbarkeit von Verhalten ausgeübt werde.

Der vorliegende Artikel untersucht, wie die spezifische soziale Ordnung, die in Kunstmuseen beobachtbar ist, von den Besuchern fortlaufend hervorgebracht wird. Sie ist nicht das Resultat der Befolgung von institutionellen Regeln, sondern entwickelt sich in und durch die Produktion von Handlungen und Interaktionen, die für andere Besucher sichtbar und nachvollziehbar ausgeführt werden (s.a. Trondsen 1976). Die Untersuchung ist Teil einer Reihe von Forschungsprojekten, die wir in den vergangenen Jahren durchgeführt haben, um ein Verständnis davon zu entwickeln, wie Besucher Ausstellungsstücke in Museen und Galerien betrachten, benutzen und verstehen (Heath/vom Lehn 2004; vom Lehn/Heath/Hindmarsh 2001). Die Projekte basieren auf ethnografischer Forschung, deren Hauptdatenquelle Videoaufnahmen von Besuchern in Museen sind. Insgesamt haben wir etwa 800 Stunden Videodaten erhoben, die jeweils von Feldbeobachtungen begleitet wurden. Hinzu kommen informelle Interviews mit dem Management der Museen sowie mit einer kleinen Anzahl von Besuchern. Die Daten, die in diesem Artikel betrachtet werden, sind in den vergangenen sie-

3) Für eine Übersicht zum aktuellen Stand der Methodenentwicklung in der qualitativen Videoanalyse vgl. auch: Knoblauch, Schnettler, Raab, Soeffner (Hg, 2006) Video-Analysis - Methodology and Methods. Qualitative Audiovisual Data Analysis in Sociology, Frankfurt am Main: Lang (im Druck). 
ben Jahren in verschiedenen Kunstmuseen und -galerien gesammelt worden. Sie zeigen Besucher unterschiedlicher soziodemografischer Herkunft, die allein oder mit Freunden, Bekannten oder Verwandten ins Museum gekommen sind - Individuen genauso wie Paare, kleine Gruppen und Schulklassen.

Den Zugang zu den Ausstellungen haben wir mit dem jeweiligen Ausstellungsmanagement ausgehandelt. Wir haben mit ihnen unser Forschungsinteresse diskutiert sowie die Praktikalitäten der Datenerhebung und ethische Aspekte video-basierter Forschung besprochen. Aus forschungsethischen Gründen ist es notwendig, das Publikum über die laufende Forschungsarbeit in Kenntnis zu setzen. Wir stellen an den Eingängen zu den Ausstellungsräumen Plakate auf, die die Besucher über die laufende Forschungsarbeit und das Filmen in den Galerien informieren. Die Plakate schildern das Ziel des Forschungsprojektes und sichern zu, dass die Videoaufnahmen ausschließlich für Forschungs- und Lehrzwecke verwendet werden. Es wird zudem darauf hingewiesen, dass Besucher jederzeit das Museumspersonal oder den Forscher, der sich in der Ausstellung befindet, darum bitten können, die Kamera abzuschalten oder aufgenommene Daten zu löschen. Bisher sind nur wenige Besucher auf uns zu gekommen, um näheres über das Projekt zu erfahren; kein Besucher hat sich geweigert, an der Untersuchung teilzunehmen.

Die Skepsis gegenüber Videoaufnahmen als sozialwissenschaftliches Datenmaterial rührt häufig daher, dass angenommen wird, die Gegenwart von Kameras in sozialen Situationen führe zwangsläufig zu einer Reaktivität der Forschungssubjekte. Die gefilmten Teilnehmer würden zu schauspielhaften Darstellungen verleitet, so dass die Reliabilität der Daten nicht gewährleistet sei; hier werden häufig Erfahrungen aus der Anthropologie herangezogen (Prosser 1998). Die häufig als methodologisches Grundsatzproblem diskutierte Reaktivität erscheint jedoch angesichts unserer praktischen Forschungserfahrung in einem ganz anderen Licht. $^{4}$

In der Besucherforschung werden schon seit langem Film- und Videoaufnahmen zu Forschungszwecken verwendet. Dabei zeigt sich, dass die Aufzeichnung von Besucherverhalten mit technischen Geräten den natürlichen Ablauf von Handlungen weitaus weniger beeinflusst als Feldbeobachtungen, die von einem Forscher vorgenommen werden, der sich in der Ausstellung aufhält (Menninger 1991; Phillips 1988; Shettel 1966). Diese Beobachtung stimmt mit Goffmans (1981: 3) Feststellung überein, dass in sozialen Situationen all diejenigen einen Status als Teilnehmer haben, die sich in wahrnehmbarer Reichweite von Ereignissen aufhalten. Als Feldforscher sind wir uns freilich bewusst, dass auch uns ein Teilnehmerstatus in der sozialen Situation am Ausstellungsstück zukommt, wenn wir uns in wahrnehmbarer Reichweite zu ihm aufhalten. Daher treffen wir Vorkehrungen, um unseren Einfluss auf die soziale Situation in Ausstellungen zu minimieren. Wir benutzen in der Regel ein oder zwei konventionelle digitale Videokameras für die Datenerhebung. Die Kamera(s) werden auf einem Stativ montiert oder an einer Wand im Ausstellungsraum angebracht, von woaus sie das Geschehen an ausgewählten Ausstellungsstücken aufzeichnen können. Die Auswahl der Ausstellungsstücke basiert einerseits auf unseren analytischen Interessen und andererseits auf dem Interesse des Museumsmanagements. Der Forscher steht nicht hinter der Kamera, sondern beobachtet die Ereignisse in der Ausstellung von einem anderen, vom Ausstellungsobjekt und Kamera entfernten Punkt aus und kehrt nur zur Kamera zurück, um das Aufnahmeband zu wechseln (Goodwin 1994).

4) Hinsichtlich der Bedeutung von Reaktivität in video-basierter Forschung und Untersuchungen, die auf Tonaufnahmen basieren siehe auch: (Laurier/Philo im Druck; Smith/McPhail/Pickens 1975; Speer/Hutchby 2003). 
Die Anwesenheit des Forschers ist aber aus noch einem anderen Grund wesentlich: Videoaufnahmen geben unweigerlich ein selektives Bild von Ereignissen wieder. Während sie eine detaillierte Analyse von Handlungen und Interaktionen erlauben, ist es nützlich zu wissen, was in Bereichen geschieht, die sich nicht vor dem Auge der Kamera abspielen. Hierzu sind Feldbeobachtungen und -notizen in den Ausstellungen ein wichtiges Mittel. Zudem sammelt der Forscher andere Materialien, wie Objektbeschriftungen und Kataloge, fertigt Skizzen des Ausstellungsaufbaus an und führt informelle Interviews mit Besuchern und Museumspersonal durch. Die Feldnotizen und die erhobenen Zusatzmaterialien bieten dem Forscher nützliche Informationen, die er später bei der Analyse der Videodaten als zusätzliche Ressource heranziehen kann, um die Handlungen der Besucher zu verstehen.

Die Analyse stützt sich hauptsächlich auf die Videodaten als primäre Datenquelle. Sie geht fallweise vor und schließt die detaillierte Analyse von bestimmten Handlungen ein, die hinsichtlich des unmittelbaren Kontextes und der interaktionalen Umgebung ausgeführt werden. Dazu werden genaue Transkripte der Gespräche und der körperlichen Handlungen der Teilnehmer und ihrer Einbettung in den interaktionalen Kontext angefertigt (Goodwin 1981). Indem Handlungen und Interaktionen, die in unterschiedlichen Fragmenten gefunden werden, miteinander verglichen werden, beginnt die Analyse schrittweise Handlungsmuster zu identifizieren. 5

In dem hier vorliegenden Artikel soll es nun darum gehen, zu untersuchen, wie Besucher in Interaktion mit all denjenigen, die sich in der gleichen sozialen Situation befinden, die Betrachtung von Kunstwerken vollziehen. Die hier vorgestellte Videoanalyse untersucht die Frage, wie Besucher ihre Navigation von Ausstellungen sowie die Betrachtung von Kunstwerken durch verbale und körperliche Handlungen miteinander koordinieren. Dabei wird ebenso die Interaktion zwischen Besuchern betrachtet, die gemeinsam ins Museum gekommen sind, wie die zwischen Besuchern, die in der gleichen Umgebung aufeinander treffen, ohne jedoch in persönlicher Beziehung zueinander zu stehen.

Die in diesem Artikel analysierten Videofragmente wurden in den Courtauld Galerien, dem Victoria und Albert Museum (beide in London) und der Djanogly Art Galerie in Nottingham aufgenommen. Wie in ethnografischen Untersuchungen üblich wurden für die Veröffentlichung Fragmente ausgewählt, die interessante und besonders klare Beispiele für die Themen und Fragestellungen darstellen, die hier zur Disposition stehen.

\section{Betrachtungsräume konfigurieren}

Besucher von Museen betrachten Kunstwerke in aller Regel in sozialen Situationen. Sie erkunden Ausstellungen gemeinsam mit Freunden, Bekannten oder Verwandten und treffen dort auf andere Besucher. Sie gehen gemeinsam mit ihren Begleitern an den Werken entlang und stoppen hier und da, um ein Exponat zu betrachten. Wenn sie durch die Ausstellung gehen, befinden sich dort auch andere Besucher, die sich ebenfalls Kunstwerke ansehen. Betrachten wir dazu den folgenden Videoausschnitt.

Das Fragment wurde in den Courtauld Galerien aufgenommen. Es beginnt, als sich zwei Besucher, Max und Flora, am hinteren Ende der Galerie mit einer der Zeichnungen beschäftigen. Beide Teilnehmer stehen leicht vorgebeugt mit gesenktem Kopf vor dem Ausstellungsstück und lesen dessen Beschriftung. Die Stellung ihrer Füße zeigt ihre projizierte Gehrichtung durch die Ausstellung an; Max steht nahezu seitlich zur Zeichnung, seine Füße

5) Weitergehende, detaillierte Darstellungen der video-basierten Ethnografie finden sich in (Bergmann 1980; Eberle 1997; Goodwin 1981, 1994; Have 1998; Heath 2004; Knoblauch 2001; vom Lehn/Heath im Druck). 
zeigen nach rechts, Floras Füße stehen in einem 45-Grad Winkel zueinander, wobei ihr rechter Fuß zum vorderen Ende der Ausstellung deutet (Bild 1.1.). Nach etwa einer Minute haben sich Max und Flora vom hinteren Ende der Galerie bis etwa in deren Mitte vorbewegt. Sie sehen sich die dort hängende Zeichnung an und lesen anschließend das dazugehörige Label. Neben ihnen steht eine Frau, die eine andere Zeichnung betrachtet. Einige Augenblicke später bewegt sich die Frau weiter nach rechts, wo sie ein Gedicht liest, das auf eine Texttafel gedruckt ist. Rechts neben ihr befindet sich eine kleine Besuchergruppe, die sich ein Ausstellungsstück in der Ecke der Galerie ansieht (Bild 1.2.).

Bild 1.1.

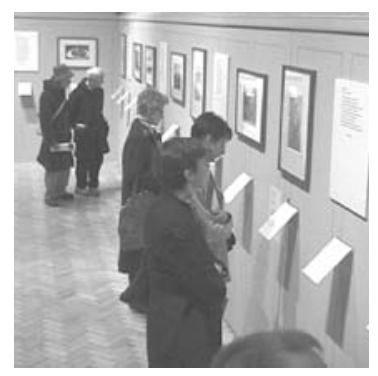

Bild 1.2.

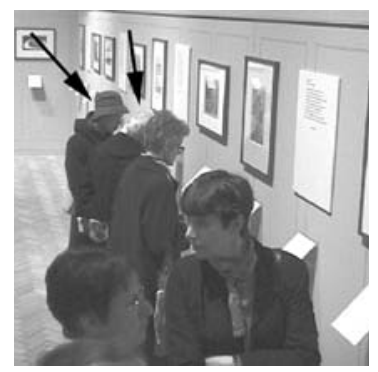

Bild 1.3.

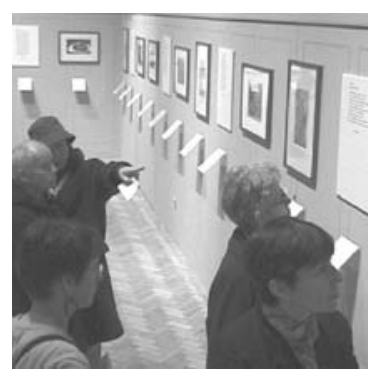

Als sich Max und Flora zum vorderen Ende der Galerie bewegen wollen, steht die Frau immer noch vor dem Gedicht, das nun auch Floras Interesse auf sich zieht. Sie deutet mit ihrem linken Arm zur Texttafel, bevor sie sich gemeinsam mit Max aus diesem Bereich der Ausstellung entfernt (Bild 1.3.).

Das Fragment zeigt, dass Besucher die Erkundung der Ausstellung und die Betrachtung der Werke mit ihren Begleitern und anderen Besuchern koordinieren. Besucher, die mit Begleitern in ein Museum gekommen sind, bewegen sich gemeinsam durch die Ausstellung und arrangieren ihre Positionen vor den Kunstwerken so, dass sie sie gemeinsam inspizieren können. Durch ihre Position, Körperhaltung und Blickrichtung konfigurieren sie zwischen sich und dem Artefakt einen Betrachtungsraum ${ }^{6}$, der der von Kendon (1990b) als »f-formation« bezeichneten räumlichen Anordnung von Interaktionsteilnehmern in zentrierter Interaktion von Anwesenden ähnelt. Der Fokus der Interaktion, der auf dem Ausstellungsstück liegt, wird durch die Körperhaltung und Blickrichtung der Teilnehmer angezeigt. Er ist jedoch keineswegs für die Dauer der sozialen Interaktion auf das Kunstwerk fixiert, sondern wird fortlaufend durch die Handlungen der Teilnehmer transformiert: In einem Augenblick betrachten sie das Kunstwerk, im nächsten das Label, dann wiederum orientieren sie sich zum Werk, bevor sie sich abwenden und zu einem anderen Objekt weitergehen.

Die Interaktionsordnung, die durch die Koordination der Handlungen der Besucher vor Kunstwerken entsteht, kann mit Knoblauch (1998) als »pragmatische Ästhetik« bezeichnet werden. Die Interaktionen vor dem Werk werden ästhetisch gestaltet, jedoch nicht um, wie etwa ein Tanz oder eine Theateraufführung, selbst einer Wertschätzung zu unterliegen, sondern, um eine alltägliche Aufgabe koordiniert zu bewerkstelligen - die Betrachtung eines Kunstwerkes. Die ästhetische Gestaltung der Interaktion vor den Kunstwerken ist für Beobachter sichtbar, für die Teilnehmer selbst jedoch unbedeutend, gilt ihr Augenmerk doch dem aktuellen Vollzug der Kunstbetrachtung.

6) Siehe hierzu auch Goffmans Beschreibung des »use space« (1971: 34-35). 
Beenden die Teilnehmer die Betrachtung eines Werkes, löst sich die Interaktionsordnung nicht in zufälliger Weise auf, sondern wird transformiert und in eine neue Form der Interaktion überführt. Die Teilnehmer gehen gemeinsam, häufig ohne sich darüber verbal zu verständigen, $\mathrm{zu}$ einem anderen Ausstellungsstück und konfigurieren dort einen neuen Betrachtungsraum. Im vorliegenden Fall steht eine Frau vor der Texttafel mit dem Gedicht, für das sich auch Flora und Max interessieren; auch die darauf folgenden Ausstellungsstücke in der vorderen Ecke der Galerie sind im Moment von anderen Besuchern besetzt. Flora und Max wenden sich um und inspizieren Zeichnungen, die auf einem Tisch in der Mitte des Ausstellungsraumes gezeigt werden. Während sich die beiden Besucher am Tisch aufhalten, bleiben sie sensibel für Handlungen und Ereignisse in ihrer unmittelbaren Umgebung. Als sich einige Augenblicke später zunächst die kleine Besuchergruppe von den Zeichnungen und dann die Frau vom Gedicht an der Wand wegbewegen, kehren Flora und Max dorthin zurück und inspizieren die Objekte (Bild 1.4. \& 1.5.).

Bild 1.4.

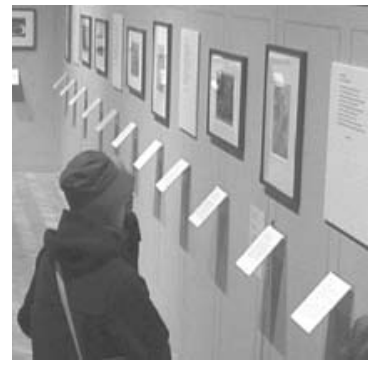

Bild 1.5.

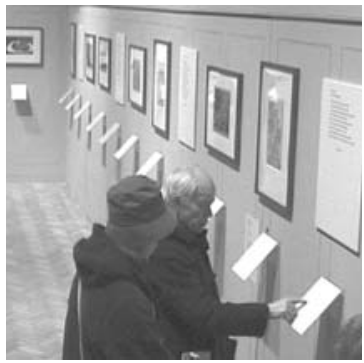

Diese Beobachtungen führen vor Augen, wie durch die soziale Organisation der Handlungen ein nahtloser Ablauf von Aktivitäten möglich wird, der gewährleistet, dass Besucher, die gleichzeitig in einer Galerie handeln und interagieren, die Ausstellung erkunden und Objekte betrachten können, ohne sich von anderen gestört oder bedrängt zu fühlen. Durch ihre verbalen und körperlichen Handlungen vollziehen sie nicht nur die Erkundung der Ausstellung, sondern sie zeigen auch ihre momentane und antizipierte Orientierung auf die Kunstwerke an. Ihre Handlungen sind »Dokumente (Garfinkel 1967), die anderen Teilnehmern als Ressourcen dienen, um ihre Aktivitäten auszuführen und in den situativen Handlungsablauf einzubetten. Die Sichtbarkeit der pragmatischen Ästhetik der Interaktion vor Kunstwerken erlaubt es Besuchern, ihre Handlungen und Interaktionen in Ausstellungen miteinander zu koordinieren - häufig ohne dabei ein Wort zu verlieren.

Analysieren wir einen weiteren Videoausschnitt, um einen anderen Aspekt der pragmatischen Ästhetik offen zu legen. Dieses Fragment wurde in der Djanogly Art Galerie in Nottingham aufgenommen, in der zur Zeit der Datenerhebung eine Serie von Drucken eines britischen Künstlers ausgestellt war. Das Fragment beginnt, als eine Besucherin - bezeichnen wir sie als Rosy - einen der Drucke betrachtet. Sie steht in einer Distanz von etwa zwei Metern vor dem Kunstwerk (Bild 2.1.). Nach etwa einer halben Minute taucht eine zweite Person - Mary - im Rücken von Rosy auf und bewegt sich um sie herum (Bild 2.2.). Sie erreicht die Höhe von Rosy, wendet sich dann nach links und beginnt Rosys Sichtlinie zu durchkreuzen (Bild 2.3.). 
Bild 2.1.

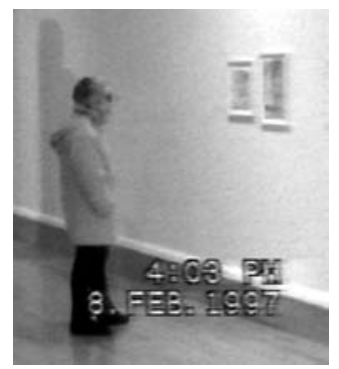

Bild 2.2.

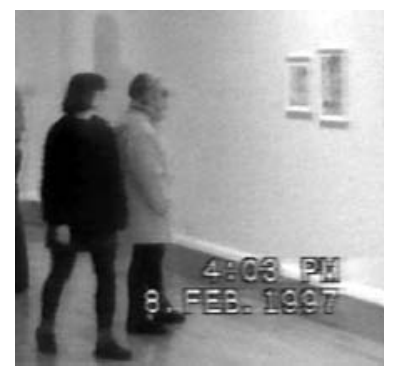

In dem Moment, in dem sie durch Rosys Sichtlinie geht, beugt sie ihren Oberkörper leicht vor (Bild 2.4.) und bewegt sich dann weiter zu dem kleinen Label, das an der Wand links neben dem Druck angebracht ist (Bild 2.5.).

Bild 2.3.

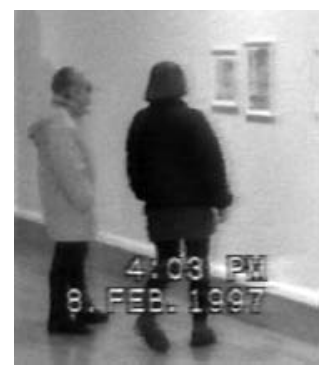

Bild 2.4.

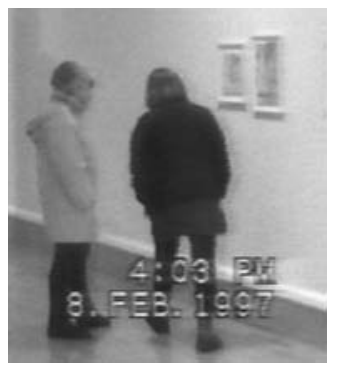

Bild 2.5.

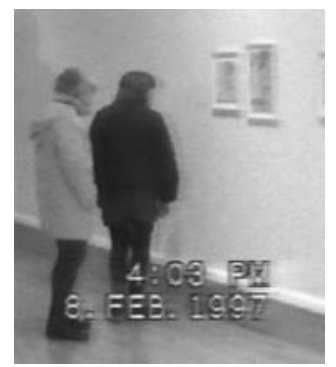

Das Fragment zeigt, dass Besucher durch ihre Handlungen die Betrachtung eines Kunstwerkes darstellen und dabei für die Handlungen anderer Besucher in der unmittelbaren Umgebung sensibel bleiben. Die Orientierung zu einem Kunstwerk ist für andere Teilnehmer sichtbar und wird von ihnen als Engagement mit einem Werk gesehen. Wenn, wie im hier dargestellten Fragment, ein Besucher - Mary - den Betrachtungsraum eines anderen - Rosy - >verletzt<, stehen ihr eine Reihe von verbalen und körperlichen Handlungen zur Verfügung, um diesem Umstand Rechnung zu tragen. Ein körperlicher Ausdruck, wie das kurze Senken des Oberkörpers (Bild 2.4.), ist beispielsweise eine Handlung, die normalerweise als Entschuldigung akzeptiert wird. In einigen Fällen wird ein solcher körperlicher Ausdruck noch von kurzen verbalen Äußerungen wie »sorry« oder »excuse me« begleitet. $^{7}$ Eine solche $>$ Entschuldigungshandlung< erlaubt es den Besuchern, ihre jeweiligen Handlungsverläufe nahtlos fortzuführen, ohne eine weitere sichtbare Reaktion zueinander zu zeigen. Rosys Reaktion auf Marys Handlung besteht sozusagen darin, keine Reaktion zu zeigen und weiterhin den Druck zu betrachten und Mary wendet sich dem Label zu, ohne in eine weitere Interaktion mit Rosy zu treten.

Wir sehen in den beiden hier analysierten Fragmenten die Bedeutung der pragmatischen Ästhetik für die Möglichkeit der relativ ungestörten Betrachtung von Kunstwerken. Durch die Art und Weise, wie Besucher vor Kunstwerken stehen und diese betrachten, erzeugen sie den Betrachtungsraum. Sie vollziehen praktische Handlungen und stellen durch eben diese Praktiken ihre Orientierung zum Kunstwerk sichtbar dar. Die sichtbare Darstellung ihrer Orientierung ist Basis für den nahtlosen Ablauf der Handlungen und Interaktionen, durch die

7) Siehe hierzu auch Goffmans Konzept von »body idiom« und »body gloss« (1971: 160). 
Besucher Museen erkunden und erfahren. Sie erlaubt es Besuchern, ihre Handlungen miteinander zu koordinieren. Sie passen die Gehgeschwindigkeit wie auch die Verweildauer an Kunstwerken an den Verlauf der Aktivitäten anderer Teilnehmer an; und sie können den Verlauf von Aktivitäten an Körperhaltungen und -handlungen anderer ablesen. Wenn ein Besucher beispielsweise ein Kunstwerk allein betrachten will, verschafft er sich den nötigen »Ellenbogenraum « (Hughes 1958), indem er seine Handlungen so vollzieht, dass die konzentrierte Betrachtung des Objektes für andere Besucher als solche sichtbar wird. Sie können an der Körper- und Kopfhaltung sowie der Art des Blickes unterschiedliche Formen des Schauens und Sehens ablesen sowie erkennen, ob der Betrachter in Kürze das Werk verlassen wird. Dadurch ist es Besuchern möglich, auch in relativ stark frequentierten Ausstellungen eine nahtlose und ungestörte Erfahrung der Werke zu haben.

\section{Kunstwerke in Interaktion betrachten}

Theorien der Kunstwahrnehmung gehen häufig davon aus, dass die Wahrnehmung von Gemälden und Skulpturen durch die Struktur der Werke bestimmt wird. In Museen betrachten und inspizieren Besucher Kunstwerke jedoch oft mit Begleitern. Sie stehen vor den Kunstwerken und verständigen sich darüber, wie sie die Objekte sehen. Das folgende Fragment, das wiederum in den Courtauld Galerien aufgenommen wurde, beleuchtet diesen interaktiven Verständigungsprozess. Es beginnt, als eine junge Frau - Petra - ein Gemälde von Rubens betrachtet, das die Familie Jan van Brueghels dem Älteren zeigt (Bild 3.1.). Als sie sich von dem Gemälde abwendet, kommt eine ältere Frau - Eva - hinzu, die sich das Gemälde zuvor etwa eine Minute lang angesehen und das dazugehörige Label gelesen hat. Eva deutet mit einer Zeigegeste auf das Gemälde und stellt dar, wie sie das Kunstwerk sieht (Bild 3.2.), »I like that (.) Thats Rubens with the Brueghel family «. ${ }^{8}$

\section{Transkript 1}

Eva: I like that (.) Thats Rubens with the Brueghel family

They are ( ) before photographs were there

(.6)

to bring them to life

another painting you see (.) pai:nted

Petra: Yah

Bild 3.1.

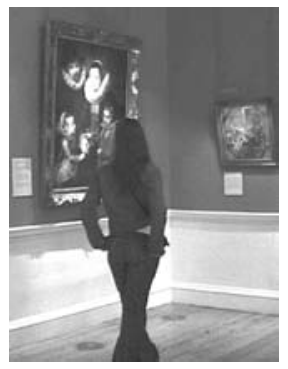

Bild 3.2.

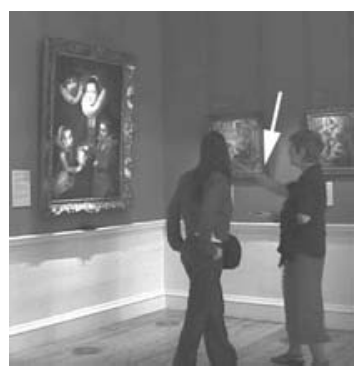

Eva: Thats Rubens...
Bild 3.3.

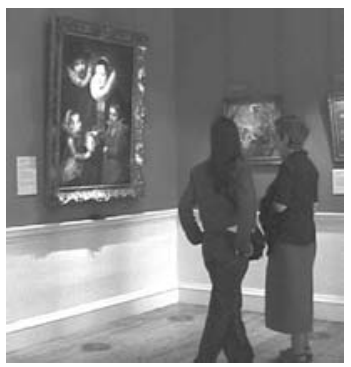

Petra: Yah

8) Kursiv gedruckte Worte und Sätze sind Ausschnitte aus Transkripten der Gespräche der Teilnehmer vor den Kunstwerken. Die Transkriptionssymbole entsprechen denen, die von Jefferson (1984) entwickelt wurden. 
Sie weist dabei insbesondere auf die Lebensechtheit der Figuren im Gemälde hin. Evas Sichtweise auf das Werk von Rubens wird von Petra mit einem kurzen »yah« bestätigt (Bild 3.3.).

Das Fragment zeigt einerseits, wie Museumsbesucher durch Körperbewegungen, verbale Äußerungen und Gesten den Betrachtungsraum konfigurieren, in dem sie ein Kunstwerk ansehen. Darüber hinaus verdeutlicht die Analyse, wie Besucher versuchen, ihre Sichtweise von Kunstwerken miteinander abzustimmen und zu koordinieren. Eva erkennt, dass Petra im Begriff ist, ihre Betrachtung des Gemäldes zu beenden. Sie tritt an Petra heran und vollzieht eine Zeigegeste begleitet vom ersten Teil ihrer Äußerung. Dadurch veranlasst sie Petra dazu, stehen zu bleiben und sich zum Kunstwerk zu drehen. Beide Teilnehmer stehen nun nebeneinander und betrachten das Gemälde.

Eva komplettiert dann ihre Äußerung und bringt dabei ihre Kenntnis des Gemäldes zum Tragen. Sie hat offenbar den Eindruck gewonnen, dass das Gemälde Rubens und die Familie von Brueghel zeigt und ist von der Lebensechtheit des Portraits beeindruckt. Durch ihre Äußerung und Gesten legt sie ihrer Begleiterin nahe, das Gemälde in einer bestimmten Art und Weise zu sehen, die Petra durch ein kurzes »yah« bestätigt. Wie die beiden Teilnehmer das Gemälde in diesem Moment sehen, ist nicht durch die Struktur des Kunstwerkes bestimmt, sondern entwickelt sich in und durch die Interaktion zwischen ihnen.

Die Verständigung über die Art und Weise, wie ein Kunstwerk gesehen wird, geht häufig über einen bloßen Verweis auf das Objekt hinaus. Durch ihre verbalen und körperlichen Handlungen stellen Teilnehmer dar, wie sie Eigenschaften eines Werkes sehen. Betrachten wir dazu ein kurzes Beispiel. Das Victoria und Albert Museum zeigt in seiner Ausstellung 〉Europe 1500-1600< Vincenzo Fogginis Skulptur »Samson und die Philister«. Die Skulptur steht auf einem Sockel, der ungefähr einen Meter und zwanzig Zentimeter hoch ist (Bild 4.0.). Im folgenden Fragment betrachten zwei Besucher, Anne und Gulia, die Front der Skulptur und lesen das am Sockel angebrachte Label, bevor Anne zu einem Ausstellungsstück hinter der Skulptur weitergeht und Gulia die Füße der Skulptur inspiziert.

Bild 4.0.

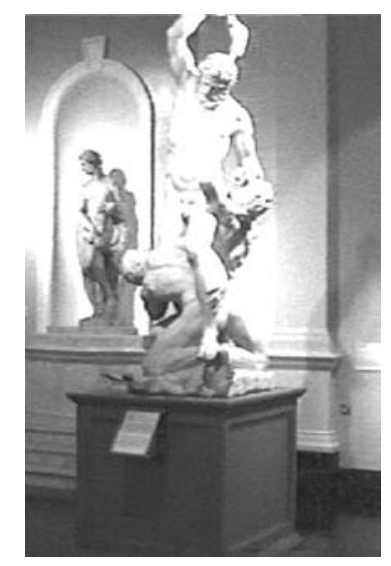

Nach wenigen Augenblicken sagt Gulia deutlich hörbar, »(But I mean)you get a lo:t of«, gefolgt von einer Pause von mehr als einer Sekunde. Während dieser Pause dreht Anne sich auf der Stelle um und orientiert sich in Richtung ihrer Begleiterin, die nun ihre Hand zu einer Zeigegeste geformt auf einen der Füße der Skulptur gerichtet hält (Bild 4.1.). 
Bild 4.1.

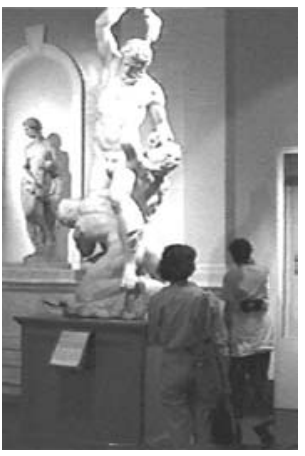

Bild 4.2.

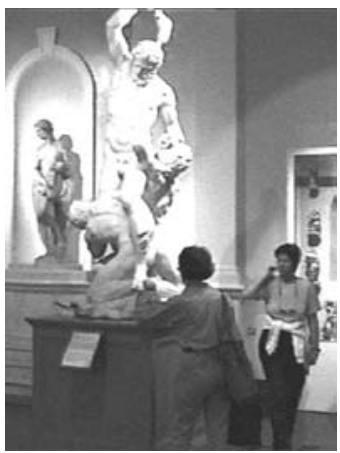

Bild 4.3 .

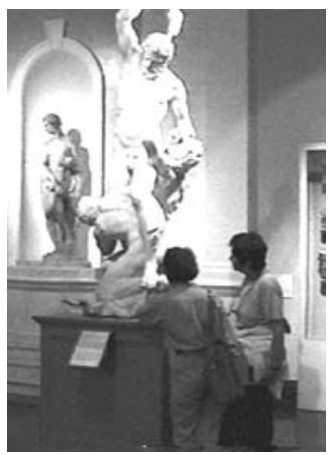

G: (But I mean)you get a lo:t of

\section{G: a lo:t of veins \\ $\mathrm{G}$ : the veins in the feet the toenails}

Wenige Augenblicke später steht Anne neben Gulia, die nun ihre begonnene Äußerung vervollständigt, »a lo:t of veins«, wodurch sie ihrer Begleiterin ihre Sichtweise auf die Skulptur anbietet. Gulia fährt dann mit der Illustration ihres Erlebnisses fort, indem sie auf die feine Gestaltung der Venen in den Füßen hinweist. Sie sagt, »the veins in the feet«, und, »the toenails«, während sie gleichzeitig eine Geste mit dem kleinen Finger ihrer linken Hand formt und an den Venen und Fußnägeln der Skulptur entlangführt (Bild 4.1. - 4.3.).

Das Fragment illustriert, wie Gulia und Anne ihre körperliche und visuelle Orientierung schrittweise miteinander koordinieren, und dadurch den Betrachtungsraum an der Skulptur konfigurieren, in dem sie einen bestimmten Aspekt der Skulptur betrachten. Gulias anfängliche Äußerung zieht Annes Aufmerksamkeit auf einen bestimmten Aspekt der Skulptur; sie vollzieht sie so, dass ihre Begleiterin Zeit hat, sich neben sie an das Ausstellungsstück zu stellen, von wo sie der mit verbalen und körperlichen Mitteln vollzogenen Beschreibung der Füße der Skulptur folgen kann. Gulia gestaltet anschließend die Beschreibung der Skulptur derart, dass sie ihre Charakteristika durch Gesten, Äußerungen und Stimmlage verkörpert; sie benutzt eine gesenkte Stimme und transformiert ihre Geste von einer flachen Hand, die über den Fuß streicht, zu einem kleinen Finger, der an den Details des feingeformten Fußes entlangfährt, so dass die Venen in den Füßen und die Fußnägel als relevante Aspekte hervorgehoben werden.

Die zwei in diesem Abschnitt analysierten Fragmente zeigen, wie Besucher Kunstwerke in Interaktion betrachten. Sie gestalten ihre verbalen Äußerungen, körperlichen Handlungen und Gesten so, dass sichtbar wird, welchen Aspekt des Kunstwerkes sie betrachten und wie sie ihn sehen. Gesten werden mit den verbalen und körperlichen Handlungen der Teilnehmer koordiniert, so dass das Objekt Teil der laufenden Interaktion wird. Dabei verkörpern die Gesten, wie das Objekt in der laufenden Situation gesehen wird. Gleichzeitig werden die Gesten so gestaltet, dass der Fokus der Interaktion nicht auf ihnen liegt, sondern auf dem Objekt, das sie verkörpern. 


\section{Kunstbetrachtung im Vorbeigehen}

Es wird zuweilen argumentiert, dass Besucher Ausstellungsstücke nur erfahren und von ihnen lernen können, wenn sie an ihnen stehen bleiben und dort eine gewisse Zeit verweilen (Bitgood 1994; Serrell 1992, 1998). Beobachtungen in Museen zeigen, dass Besucher zwar nicht an allen Kunstwerken stehen bleiben und sie betrachten, doch sehr wohl Kunstwerke ansehen, während sie an ihnen vorbeigehen. Sie erfahren also nicht nur die Ausstellungsstücke, an denen sie stoppen, sondern erzeugen den Kontext, in dem sie Kunstwerke wahrnehmen, indem sie Gehen und Betrachten miteinander koordinieren (vom Lehn/Heath/Hindmarsh 2001). Während Besucher durch eine Ausstellung gehen, sehen sie auch andere Besucher, die vor Kunstwerken stehen und sie betrachten. Untersuchen wir dazu das folgende Fragment, das wiederum in den Courtauld Galerien in London aufgenommen wurde.

Es beginnt, als zwei Frauen, Maria (rechts) und Paula (links), an der linken Seite von Edouard Manets >Un Bar aux Folies-Bergère< stehen und das Label lesen, das an der Wand links vom Gemälde angebracht ist. Während sie dort stehen, bewegt sich ein junger Mann durch die Ausstellung auf ihre Position am Gemälde zu (Bild 5.1.).

Bild 5.1.

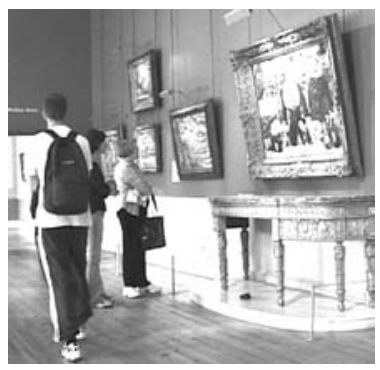

Bild 5.2.

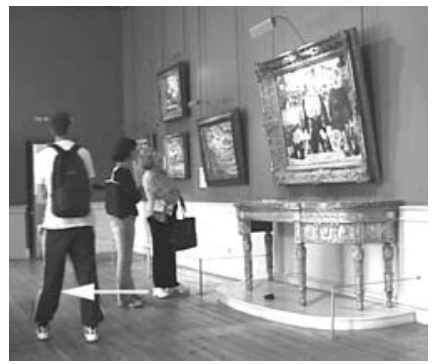

Wenige Augenblicke später schaut Maria auf und betrachtet das Gemälde, während Paula fortfährt, das Label zu lesen. Der Mann, der mittlerweile in der Höhe des Bildes angelangt ist, dreht seinen Kopf leicht nach rechts in Richtung des Gemäldes und macht einen Schritt nach links, wodurch er den Abstand zu den anderen Besuchern sichtbar vergrößert (Bild 5.2.). Als Maria mit einer deutlich sichtbaren Zeigegeste auf den oberen Bereich von Manets Werk deutet und sagt, »Ah I've seen this one before«, dreht er seinen Kopf weiter zum Gemälde hin (Bild 5.3.). Diese Geste und möglicherweise die verbale Äußerung veranlassen den Mann, seinen Blick zu dem Bereich des Gemäldes zu wenden, auf den die Geste der Frau deutet (Bild 5.4.).

Bild 5.3.

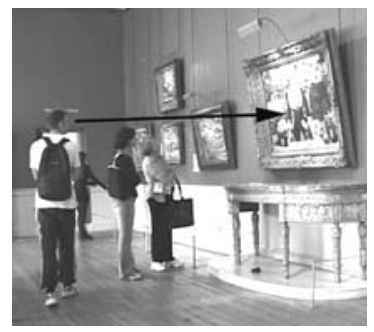

Bild 5.4.

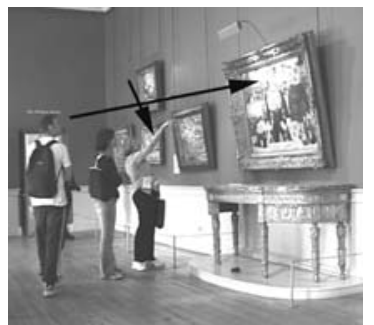

Maria: Ah I've seen this one before
Bild 5.5.

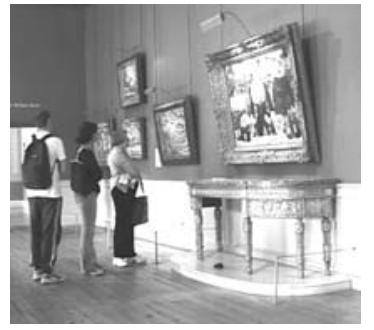


Für ihn wird ein Aspekt des Gemäldes durch Interaktionen von Besuchern sichtbar und relevant, mit denen er keine andere Beziehung hat, als dass sie in seiner wahrnehmbaren Reichweite miteinander interagieren. Obwohl er die Ausstellung ohne Begleiter erkundet, sieht er das Gemälde nicht unabhängig von anderen, sondern im Licht der Interaktion von Besuchern, die es gemeinsam anschauen, wenn er dort vorbeigeht. Einen Moment später wendet er seinen Blick von Manets Gemälde ab und schaut in Richtung des nächsten Ausstellungsstückes, das links neben den beiden Frauen an der Wand angebracht ist (Bild 5.5.).

In Museen ist die Betrachtung von Kunstwerken ein »beobachtbares und beschreibbares Phänomen« (Garfinkel 1967), also ein Dokument ihrer Orientierung zum Werk. Die Betrachtung eines Kunstwerkes kann daher für all jene relevant werden, die sich in wahrnehmbarer Reichweite aufhalten. Während sie an einem Objekt vorbeigehen, das von anderen Besuchern betrachtet wird, sehen sie nicht nur die Betrachter und das Werk, sondern sie sind auch sensibel dafür, welche Aspekte des Werkes sich die Betrachter ansehen und wie sie es betrachten. Museumsbesucher können an den Handlungen des Betrachters beispielsweise ablesen, ob er das Kunstwerk aus der Nähe inspiziert oder es sich nur beiläufig ansieht, oder auch ob vor dem Kunstwerk eine wahrnehmbare Reaktion hervorgebracht wird. Die Kunstbetrachtung wird in diesen Fällen sozusagen durch die Handlungen und die Interaktion vermittelt, die andere Teilnehmer vor dem Werk ausführen.

Die Analyse des Fragments hilft uns, die Vermittlung von Kunsterfahrung durch Handlungen anderer Besucher zu verstehen. Die Besucher führen ihre Handlungen so aus, dass Blickkontakt vermieden wird und es zu keiner längeren Interaktionssequenz kommt. Die Besucher am Kunstwerk wenden dem vorbeigehenden Besucher den Rücken zu und ignorieren seine kurzzeitige Anwesenheit in ihrer lokalen Umgebung. Der vorbeigehende Besucher blickt nicht zu den anderen Teilnehmern, sondern zum Kunstwerk und dem Aspekt, mit dem sie sich beschäftigen. Er sieht das Gemälde im Lichte der Interaktion anderer Besucher vor dem Ausstellungsstück, die er als Ressource nutzt, um im Vorbeigehen einen Eindruck vom Kunstwerk zu bekommen. Dabei hält er seine Orientierung zum Werk nur solange aufrecht, wie die anderen Besucher nicht anzeigen, dass ihre Betrachtung des Objektes von seiner Gegenwart beeinflusst wird.

Zentral für die Möglichkeit der Kunstbetrachtung im Vorbeigehen ist die »temporale Organisation des Blickes « (Sudnow 1972) zum Gemälde. Würde der Mann im dargestellten Fall hinter dem Betrachtungsraum der anderen Besucher stehen bleiben und seinen Blick zwischen dem Kunstwerk und ihnen alternieren, könnte seine Handlung leicht als >Warten aufgefasst werden. Dies könnte von den anderen Besuchern als eine unausgesprochene Aufforderung verstanden werden, dem Mann Platz an diesem Ausstellungsstück einzuräumen. Derartige Handlungen, die andere direkt oder indirekt zum Weitergehen auffordern, sind in den meisten Museen im Allgemeinen nur selten zu beobachten. Beispiele hierfür finden sich hingegen in sehr populären Ausstellungen, sowie vor Ausstellungsstücken, die besonders prominent sind, wie beispielsweise die >Mona Lisa< im Pariser Louvre. Dort scheint $\gg$ Warten« und »Bedrängen« zur Normalität zu gehören, was zu einer Kritik von sogenannten Blockbuster-Ausstellungen geführt hat, die große Besuchermengen anziehen. Diese Kritik rührt wohl unter anderem daher, dass es Besuchern schwer fällt, sich den nötigen »Ellenbogenraum« zu verschaffen, so dass die Integrität ihres Betrachtungsraumes häufig von den Handlungen anderer Besucher verletzt wird. In diesen Fällen verlangt die Aufrechterhaltung des Betrachtungsraumes soviel Interaktionsarbeit, dass es den Teilnehmern schwer fällt, ihre Aufmerksamkeit auf das Werk zu richten. Dagegen markiert das von einem kurzen Blick zum Gemälde begleitete >Vorbeigehen $<$ an einem Betrachtungsraum ein Desinteresse an den 
anderen Besuchern und ihrer Interaktion. ${ }^{9}$ Es ermöglicht dem vorbeigehenden Teilnehmer gleichzeitig jedoch mehr als nur einen beiläufigen Eindruck vom Kunstwerk zu bekommen, da er das Gemälde nicht einfach nur im Vorbeigehen sieht, sondern im Licht der Interaktion anderer Besucher.

\section{Diskussion}

Theorien und Konzepte der Kunstwahrnehmung fokussieren zumeist auf den individuellen Betrachter und seine Erfahrung von Kunstwerken. Nur selten nehmen sie darauf Bezug, dass die Betrachtung von Kunstwerken in Museen normalerweise in sozialen Situationen vollzogen wird. Untersuchungen, die auf die gleichzeitige Anwesenheit mehrerer Besucher im gleichen Ausstellungsraum hinweisen, sehen die sozialen Bedingungen der Kunstbetrachtung als Störfaktor (Csikszentmihalyi/Robinson 1990). Dieser Artikel hat dagegen untersucht, wie die Betrachtung von Kunstwerken und anderen Ausstellungsstïcken in Museen dadurch ermöglicht wird, dass Besucher ihre Handlungen vor den Objekten so organisieren, dass eine Interaktionsordnung sichtbar wird, die wir mit Knoblauch (1998) als »pragmatische Ästhetik « bezeichnet haben.

In Museen werden Kunstwerke in und durch Interaktion mit anderen Besuchern betrachtet und wahrgenommen. Besucher, die sich im gleichen Ausstellungsraum aufhalten, passen ihre Gehgeschwindigkeit und Verweildauer an den Objekten systematisch aneinander an. Sie gestalten ihre Handlungen vor Kunstwerken so, dass ein Betrachtungsraum sichtbar wird, den andere erkennen können und respektieren. Sie lassen erkennen, wie sie Kunstwerke betrachten und welchen Aspekten der Objekte sie ein besonderes Interesse entgegenbringen. Die Kunstbetrachtung ist ein für andere Besucher sichtbares Dokument der Orientierung zu einem Objekt. Es kann unterschieden werden, ob ein Betrachter dem Kunstwerk nur einen kurzen Blick zuwirft, es näher betrachtet oder inspiziert. Die Unterscheidung von Betrachtungsweisen erlaubt Rückschlüsse auf die Eigenschaften des Objektes sowie auf den weiteren Verlauf der Handlungen des Betrachters. Dadurch können Besucher Eigenschaften von Kunstwerken durch die Interaktion anderer Betrachter vor den Objekten wahrnehmen und ihre Handlungen in Ausstellungen nahtlos miteinander koordinieren, so dass eine ungestörte Erfahrung der Werke möglich wird, obwohl die Teilnehmer fortwährend miteinander interagieren.

Die Analyse der Videofragmente hat die Kunstfertigkeit der Handlungsausführung und koordination der Teilnehmer herausgearbeitet. Sie gestalten ihre Körperbewegungen, Äußerungen, Gesten, und Blicke in einer Art und Weise, die einer choreografierten oder inszenierten Sequenz ähnelt und daher als ästhetisch wahrgenommen werden können. Die Handlungen werden jedoch nicht vollzogen, um von anderen in ihrer ästhetischen Ausführung gesehen zu werden, sondern sie dienen der Pragmatik der Handlungen, durch die die Kunstwerke betrachtet werden. Sie werden kunstvoll mit anderen Teilnehmern koordiniert, wodurch eine Interaktionsordnung in Ausstellungen sichtbar wird, die den nahtlosen Ablauf von Museumsbesuchen sowie die unbeeinträchtigte Betrachtung der Werke ermöglichen. Die ästhetische Ausführung und Koordination der Handlungen vor den Kunstwerken sind die Grundlage für die Möglichkeit der Betrachtung der Objekte im Museum.

Wir sehen zudem in den hier untersuchten Videofragmenten Beispiele für das direkte Aufeinandertreffen ästhetischer und pragmatischer Motive. Die Betrachtung und Kommunikation über Kunst im Museum ist von pragmatischen Motiven durchzogen (s.a. Knoblauch 1998: 323). Während die Kunstwerke ausgestellt werden, um von Besuchern unter ästhetischen Gesichtspunkten beurteilt zu werden, müssen Besucher den pragmatischen Forderungen der spezifischen Situation im Museum gerecht werden, damit sie die Werke betrachten können.

9) Goffman (1963: 83-88) hat diese Form des Desinteresses als »civil inattention« bezeichnet. 
Die Beobachtungen, die in diesem Artikel geschildert wurden, werfen ein anderes Licht auf die Kunstwahrnehmung, als sie konventionell in der Wahrnehmungspsychologie oder auch Teilen der Kunstsoziologie beschrieben wird. Sie zeigen insbesondere, dass Kunstwahrnehmung nicht durch die Struktur der Werke oder kognitive Dispositionen vorherbestimmt ist, sondern in spezifischen, lokalen und sozialen Situationen vollzogen wird. Dieser Artikel hat einige der Spezifika der Situationen, in denen Kunstwerke betrachtet werden, herausgearbeitet. Weitere detaillierte soziologische Untersuchungen sind erforderlich, um zu verstehen, wie Besucher Kunstwerke in Museen in und durch soziale Interaktion betrachten.

\section{Literatur}

Arnheim, Rudolf (2000): Kunst und Sehen. Eine Psychologie des schöpferischen Auges. Berlin \& New York, de Gryter.

Becker, Howard S. (1982): Art Worlds. Berkeley, Los Angeles \& London, University of California Press. Bennett, Tony (1995): The Birth of the Museum. History, Theory, Politics. London, Routledge.

Bergmann, Jörg (2005): Studies of Work, in: F. Rauner (Hrsg.), Handbuch der Berufsbildungsforschung, Bielefeld, Bertelsmann Verlag, 639-646.

Bergmann, Jörg R. (1980): Ethnomethodologische Konversationsanalyse, in: P. Schröder/H. Steger, Dialogforschung. Jahrbuch 1980 des Instituts für deutsche Sprache, Düsseldorf, Institut für Deutsche Sprache, 9-52.

Bitgood, Stephen (1994): Problems in visitor orientation and circulation. The Educational Role of the Museum, in: E. Hooper-Greenhill. Leicester, Leicester University Press, 64-75.

Bourdieu, Pierre (1987): Die feinen Unterschiede. Frankfurt am Main, Suhrkamp.

Bourdieu, Pierre (1991): The Love of Art, Stanford University Press.

Bruder, Kurt A. \& Ucok, Ozum (2000): »Interactive Art Interpretation: How Viewers Make Sense of Paintings in Conversation.« Symbolic Interaction 23, 337-358.

Csikszentmihalyi, Mihaly \& Robinson, Rick E. (1990). The Art of Seeing. An Interpretation of the Aesthetic Encounter. Malibu, Getty.

Doering, Nicola \& Hirschauer, Stefan (1997): Die Biographie der Dinge. Eine Ethnographie musealer Repraesentation, in: S. Hirschauer/K. Amann, Die Befremdung der eigenen Kultur. Zur ethnographischen Herausforderung soziologischer Empirie, Frankfurt am Main, Suhrkamp, 267-297.

Eberle, Thomas Samuel (1997): Ethnomethodologische Konversationsanalyse. in: R. Hitzler/A. Honer, Sozialwissenschaftliche Hermeneutik, Opladen, UTB, 245-279.

Falk, John \& Dierking, Lynn (2000): Learning from Museums. Visitor Experiences and the Making of Meaning. Walnut Creek, Lanham, New York and Oxford, Alta Mira Press.

Garfinkel, Harold (1967): Studies in Ethnomethodology, Blackwell.

Gerhards, Jürgen (Hrsg.), (1997): Soziologie der Kunst. Produzenten, Vermittler und Rezipienten. Opladen, Westdeutscher Verlag.

Goffman, Erving (1963): Behavior in Public Places. Notes on the Social Organization of Gatherings. New York and London.

Goffman, Erving (1971): Relations in Public. Microstudies of the Social Order. New York, Basic Books.

Goffman, Erving (1981): Forms of Talk. Pennsylvania.

Gombrich, E. H. (1960): Art and Illusion. New York, Pantheon.

Gombrich, Ernst H. (1982): The Image and the Eye. Further studies in the psychology of pictorial representation. London, Phaidon.

Goodwin, Charles (1981): Conversational Organization: Interaction Between Speakers and Hearers. New York, Academic Press.

Goodwin, Charles (1994): »Recording human interaction in natural settings.« Pragmatics 3, 181-209. 
Hausendorf, Heiko (2005): Die Kunst des Sprechens über Kunst, in: P. Klotz/C. Lubkoll, Beschreibend wahrnehmen - wahrnehmend beschreiben Sprachliche und ästhetische Aspekte kognitiver Prozesse. Freiburg, Rombach Druck- und Verlagshaus, 99-134.

Have, Paul ten (1998): Doing Conversation Analysis: A Practical Guide. London, Sage.

Heath, Christian (1986): Body Movement and Medical Interaction. Cambridge, Cambridge University Press.

Heath, Christian (2004): Analysing face-to-face interaction: video, the visual and material. In: D. Silverman (Hrsg.), Qualitative Research. Theory, Mehtod and Practice, London, Thousand Oaks \& New Delhi, Sage, 266-282.

Heath, Christian \& Luff, Paul (2000): Technology in Action. Cambridge, Cambridge University Press.

Heath, Christian \& vom Lehn, Dirk (2004): »Configuring Reception: (Dis-)Regarding the >Spectator $<$ in Museums and Galleries. «Theory, Culture \& Society 21, 43-65.

Hein, George (1998): Learning in the Museum. Cambridge/MA, Routledge.

Hirschauer, Stefan (2002): »Scheinlebendige. Die Verkörperung des Letzten Willens in einer anatomischen Ausstellung.« Soziale Welt 53, 5-30.

Hughes, Everett C. (1958). Men and Their Work. New York, The Free Press.

Hudson, Kenneth (1975): A Social History of Museums. New Jersey, Humanities Press.

Jefferson, Gail (1984). Transcript notation. Structures of Social Action. Studies in Conversation Analysis. J. M. Atkinson/J. Heritage. Cambridge, Cambridge University Press, ix-xvi.

Karp, Ivan \& Lavine, Steven D. (Hrsg.), (1991), Exhibiting Cultures: The Poetics and Politics of Museum Display, Smithonian Institution Press.

Kendon, Adam (1990a): Conducting Interaction. Patterns of behavior in focused encounters. Cambridge.

Kendon, Adam (1990b): Spatial Organization in Social Encounters: the F-formation system. Conducting Interaction: Patterns of behavior in focused encounters. ibid. Cabridge, New York, et al., Cambridge University Press, 209-237.

Kindt, Wolfgang (1982): »Social Functions of Communication about Works of Art.« Poetics 11, 393-418.

Klein, Hans Joachim (1996): Besucherforschung als Antwort auf neue Herausforderungen, in: Haus der Geschichte (Hrsg.), Museen und ihre Besucher. Herausforderungen in der Zukunft. Bonn, Argon Verlag, 72-84.

Knoblauch, Hubert (1998): Pragmatische Ästhetik. Inszenierung, Performance und die Kunstfertigkeit alläglichen kommunikativen Handelns, in: H. Willems/M. Jurga (Hrsg.), Inszenierungsgesellschaft. Ein einführendes Handbuch, Opladen \& Wiesbaden, Westdeutscher Verlag, 305-324.

Knoblauch, Hubert (2001): »Fokussierte Ethnographie.« Sozialer Sinn 1, 123-141.

Knoblauch, Hubert (2004): »Die Video-Interaktions-Sequenzanalyse.« Sozialer Sinn 3, 157-163.

Knoblauch, Hubert \& Heath, Christian (1999): »Technologie, Interaktion und Organisation: Die Workplace Studies. « Schweizerische Zeitschrift für Soziologie 25, 163-181.

Laurier, Eric \& Philo, Chris (im Druck): Natural Problems of Naturalistic Video Data, in: H. Knoblauch/ B. Schnettler/J. Raab/H.-G. Soeffner: Video Analysis. Methodology and Methods. Qualitative Audiovisual Data Analysis in Sociology, Frankfurt am Main, Peter Lang.

Lawrence, Ghislaine. (1993): Remembering rats, considering culture: perspectives on museum evaluation, in: S. Bicknell/G. Farmelo (Hrsg.), Visitor Studies of the 90s, London, Science Museum, 117-124.

Leinhardt, Gaea., Crowley, K. \& Knutson, K. (Hrsg.): (2002), Learning Conversations in Museums. Mahwah, NJ, LEA.

Leinhardt, Gaea \& Knutson, Karen (2004), Listening in on Museum Conversations. Walnut Creek, Altamira Press.

Loer, Thomas (1996): Halbbildung und Autonomie. Über Struktureigenschaften der Rezeption bildener Kunst. Opladen., Westdeutscher Verlag.

Luff, Paul, Hindmarsh, Jon \& Heath, Christian, (Hrsg.) (2000), Workplace Studies. Recovering Work Practice and Informing System Design. Cambridge, Cambridge University Press. 
Luhmann, Niklas (1998): Die Kunst der Gesellschaft. Frankfurt am Main, Suhrkamp.

Macdonald, S. (2002): Behind the Scenes at the Science Museum. Oxford and New York, Berg.

Menninger, Margret (1991): The analysis of time data in visitor research and evaluation studies, in: S.Bitgood/A Benefield/D. Patterson (Hrsg.), Visitor Studies: Theory, Research, and Practice, Jacksonville/ AL, Visitor Studies Association, 104-113.

Phillips, David (1988): »Recipe for an interactive art gallery.« Museum Management and Curatorship 7, 243-252.

Prosser, Jon, Ed. (1998): Image-based Research. A Sourcebook for Qualitative Researchers. London, Farmer Press.

Raab, Jürgen (2002): »>Der schönste Tag des Lebens < und seine Überhöhung in einem eigenwilligen Medium. Videoanalyse und sozialwissenschaftliche Hermeneutik am Beispiel eines professionellen Hochzeitsvideofilms.« sozialer sinn 3, 469-495.

Ramachandran, Vilayanur S./Hirstein, William (1999): »The Science of Art: A neurological theory of aesthetic experience. « Journal of Consciousness Studies 6, 15-51.

Sacks, Harvey (1992): Lectures on Conversation. Oxford, Blackwell.

Schaefer, Hermann (Hrsg.), (1996): Museen und ihre Besucher. Herausforderungen in der Zukunft. Bonn \& Berlin, Haus der Geschichte.

Schiele, B. (1993): Creative Interaction of Visitor and Exhibition, in: S.Bitgood/A Benefield/D. Patterson (Hrsg.), Visitor Studies: Theory, Research, and Practice. Vol. 5, 1. Selected Papers from the 1992 Visitor Studies Conference, Jacksonville, AL, Visitor Studies Association, 28-56.

Schnettler, Bernt (2001): »Vision und Performanz.« Sozialer Sinn 1, 143-163.

Schnettler, Bernt (2003): Methodological bases of video analysis: Visual hermeneutics and communicative genres. Paper presented at the 6th Conference of the ESA, Murcia, 23.-26.9 2003 (Ms.).

Serrell, Beverly (1992): »The 51\% solution: Defining a successful exhibit by visitor behavior.« Current Trends in Audience Research and Evaluation 6, 26-30.

Serrell, Beverly (1998): Paying Attention: Visitors and Museum Exhibitions. Washington D.C., American Association of Museums.

Shettel, Harris (1966): An Evaluation Model for Measuring the Impact of Overseas Exhibits. Pittsburgh/ Pennsylvania, American Institute for Research in the Behavioural Sciences, Pittsburgh, Pennsylvania 1966 (Ms.).

Smith, Richard L., McPhail, Clark \& Pickens, Robert G. (1975): »Reactivity to Systematic Observation with Film: A Field Experiment.« Sociometry 38(4), 536-550.

Speer, Susan A. \& Hutchby, Ian (2003): "From ethics to analytics: Aspects of participants' orientations to the presence and relevance of recording technologies." Sociology 37, 315-337.

Sudnow, David (1972): Temporal Parameters of Interpersonal Observation, in D. Sudnow (Hrsg.), Studies in Social Interaction, New York, 259-279.

Tanner, Jeremy, Ed. (2003): The Sociology of Art. A Reader. London \& New York, Routledge.

Trondsen, Norman (1976): »Social Control in the Art Museum.« Urban Life 5, 105-119.

vom Lehn, Dirk/Heath, Christian (in press). Interaction at the exhibit-face: videobased studies in museums and galleries, Video-Analysis. Methodology and Methods. H. Knoblauch and B. Schnettler. London, Peter Lang.

vom Lehn, Dirk/Heath, Christian/Hindmarsh, Jon (2001): »Exhibiting Interaction: Conduct and Collaboration in Museums and Galleries.«Symbolic Interaction 24, 189-216.

Warning, Rainer (1994): Rezeptionsästhetik. Theorie und Praxis. München, UTB.

Dirk vom Lehn, Work, Interaction \& Technology Research Group,

Department of Management, King's College London, Franklin-Wilkins Building, 150 Stamford Street, London SE1 9NH, UK.

Dirk.vom_lehn@kcl.ac.uk 